\title{
Ventricular Tachyarrhythmias in a Canine Model of LQT3 Arrhythmogenic Effects of Sympathetic Activity and Therapeutic Effects of Mexiletine
}

\author{
Masaomi Chinushi, MD; Minoru Tagawa, MD*; Hirotaka Sugiura, MD*; \\ Satoru Komura, MD*; Yukio Hosaka, MD*; \\ Takashi Washizuka, MD*; Yoshifusa Aizawa, MD
}

\begin{abstract}
The ventricular tachyarrhythmias associated with the LQT3 syndrome are typically bradycardia-dependent. However, some episodes can be associated with exercise or emotional stress, suggesting a different arrhythmogenic mechanism when sympathetic activity predominates. This study examined the potential arrhythmogenic mechanisms during periods of autonomically mediated transient heart rate acceleration in a canine anthopleurin-A model of LQT3 syndrome. Using plunge needle electrodes, transmural unipolar electrograms of the left ventricle were recorded from endocardial (Endo), mid-myocardial (Mid) and epicardial (Epi) sites. The activation-recovery interval (ARI) was measured to estimate local refractoriness. The cardiac cycle length was gradually shortened by cessation of vagal stimulation (vagal stimulation protocol (VSP)), and intramural electrograms and onset mode of ventricular tachyarrhythmias were analyzed in 7 experiments. The VSP was performed 8 times before and 5 times after administration of mexiletine in each experiment. Before mexiletine, vagal stimulation slowed the heart rate and created large transmural ARI dispersion because of a greater ARI prolongation at Mid rather than Epi/Endo sites. After cessation of vagal stimulation, unipolar electrograms started to show ARI alternans and ventricular premature beats developed sporadically. Sustained ventricular tachyarrhythmias were induced in 12 of the 56 trials of the VSP. Initiation of ventricular tachyarrhythmias was associated with delayed conduction at Mid/Endo sites. Mexiletine attenuated transmural ARI dispersion, and neither ARI alternans nor ventricular tachyarrhythmias was observed during all 35 trials of the VSP after mexiletine administration. Heart rate acceleration induced by an abrupt shift to a state of predominant sympathetic activity enhances arrhythmias in this LQT3 model. Mexiletine homogenizes ventricular repolarization, suppresses premature complexes and was antiarrhythmic during ventricular tachyarrhythmias induced by the VSP. (Circ J 2003; 67: 263-268)
\end{abstract}

Key Words: Long QT syndrome; Mexiletine; Repolarization alternans; Sympathetic activity

$\mathbf{R}$ ecent clinical and experimental studies of the congenital long QT syndrome (LQTS) have shown that the triggers of the ventricular tachyarrhythmias (VTA) and the response to therapeutic interventions vary among the genetically defined subtypes of LQTS 1,2 For example, most cardiac events in LQT1 patients are associated with increased sympathetic activity and $\beta$ blocker treatment is considered useful in preventing VTA in this group of patients ${ }^{3,4}$ On the other hand, VTA in the LQT3 syndrome are bradycardia-dependent and $\beta$-adrenergic blocker therapy appears to have limited efficacy in this population.,5 However, in recent clinical studies, 30-35\% of VTA in LQT3 patients occurred during exercise or emotional stress, suggesting an arrhythmogenetic role played by increased sympathetic activity in LQT3 as well? Although mexiletine causes a greater QT interval shortening in LQT3 syndrome than in other subtypes of LQTS,6,7 a correlation between the degree of QT interval shortening and the therapeutic effects of mexiletine has not been

(Received October 31, 2001; revised manuscript received December 6, 2002; accepted December 17, 2002)

School of Health Science and *First Department of Internal Medicine, Niigata University School of Medicine, Niigata, Japan

Mailing address: Masaomi Chinushi, MD, School of Health Science, Niigata University School of Medicine, 2-746 Asahimachi, Niigata 951-8518, Japan. E-mail: masaomi@clg.niigata-u.ac.jp established.

Using an animal model of LQTS, we focused on arrhythmogenesis in the period of heart rate acceleration induced by a shift of autonomic activity from a parasympathetic to a predominantly sympathetic state? Our recent study in a canine anthopleurin-A model of LQT3 showed that withdrawal of vagal stimulation produced gradual shortening of the cardiac cycle and VTA was sometimes induced during the transition period of the cardiac cycle length? To further study the arrhythmogenesis in the sympathetic activity predominant period, the incidence and onset mode of VTA were analyzed before and after administration of mexiletine in a new experimental setting of the canine model of LQT3. The therapeutic effect of mexiletine was assessed by its effect on intramural repolarization and the inducibility of VTA by modulation of vagal tone.

\section{Methods}

\section{Surgical Preparation}

The study was approved by the Animal Studies Subcommittee of the Institutional Review Board, and confirmed with the Guide for the Care and Use of Laboratory Animals published by the US National Institutes of Health. Experiments were performed in 7 beagles $(10.0-14.0 \mathrm{~kg})$, which were anesthetized with 
A

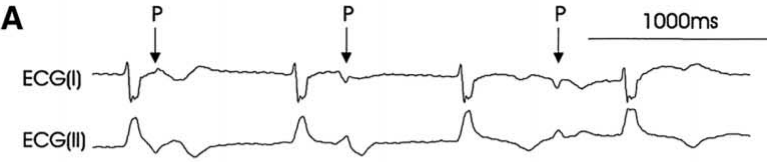

Endo

ARID
104
94

95

$$
\text { B }
$$

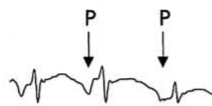<smiles>PC1(P)CCCCC1</smiles>

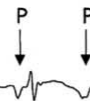

$1000 \mathrm{~ms}$ (II) $M$ Endo

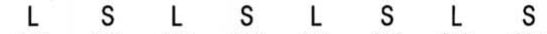
$\sqrt{347} \sqrt{230}^{234} \sqrt{347}^{345} \sqrt{3}^{233} \sqrt{230}^{233} \sqrt{342}^{234}-$

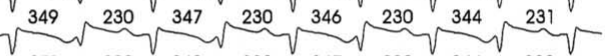
id $\sqrt{353} \sim_{227}^{228} \sqrt{350} \sim_{228}^{229} \sqrt[347]{348} \underbrace{229}_{230} \sqrt{345} \overbrace{230}^{230} \sqrt{-}$ $\sqrt{354} \sqrt{231} \sqrt{351} \sqrt{231} \sqrt{350} \sqrt{233} \sqrt{347} \sqrt{234}$ $\sqrt{352} \sqrt{235} \sqrt{349}{ }_{236}^{236} \sqrt{347} \sqrt{238} \sqrt{344} \int_{237}^{234} \sqrt{ }$

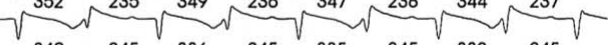
$r^{340} \sim^{245} \sim^{2436} r^{3355} r^{2432} r^{245}$

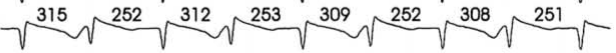

Fig 1. Intramural repolarization pattern during (A) and after cessation (B) of vagal stimulation in experiment \#3. Leads I and II of the surface ECG and the transmural unipolar electrograms are shown from top to bottom. The activation-recovery interval (ARI) is indicated with each corresponding cycle. Vagal stimulation induces a slow ventricular rhythm and creates large (94-104 ms) transmural ARI dispersion (ARID). After cessation of vagal stimulation, the heart rate gradually increases and unipolar electrograms show ARI alternans. Cycles with the longer QT intervals (L), are associated with longer ARI at the midmyocardium (Mid) than at epicardial (Epi) sites. Conversely, ARI at the Mid sites is shorter than at the Epi site when the QT interval is shorter (S), reversing the gradient of transmural repolarization. p, p wave on the surface ECG.

Table 1 Inducibility of Ventricular Tachyarrhytnmia

\begin{tabular}{cccc}
\hline \hline Experiment no. & Before mexiletine & After mexiletine & \\
\hline 1 & $2 / 8$ & $0 / 5$ & \\
2 & $1 / 8$ & $0 / 5$ & \\
3 & $1 / 8$ & $0 / 5$ & \\
4 & $2 / 8$ & $0 / 5$ & \\
5 & $2 / 8$ & $0 / 5$ & \\
6 & $3 / 8$ & $0 / 5$ & \\
7 & $1 / 8$ & $0 / 5$ & \\
Total & $12 / 56(21 \%)$ & $0 / 35(0 \%)$ & $p<0.003$ \\
\hline
\end{tabular}

sodium thiopental $(17.5 \mathrm{mg} / \mathrm{kg}$ intravenous bolus, followed by a maintenance dose of $5.0 \mathrm{mg} \cdot \mathrm{kg}^{-1} \cdot \mathrm{h}^{-1}$ ), intubated, and artificially ventilated. Catheters were inserted into the femoral vein for administration of fluids and drugs, and into the femoral artery to monitor blood pressure. Leads I, II and $\mathrm{aVF}$ of the surface electrocardiogram (ECG) and blood pressure were continuously monitored. The heart was exposed via a midline sternotomy. Core temperature was maintained constant at $37^{\circ} \mathrm{C}$ with a thermostatically controlled thermal blanket. Saline warmed to $37^{\circ} \mathrm{C}$ was regularly applied to the heart to moisten and prevent cooling of the epicardial surface. Upon completion of the experiment, ventricular fibrillation was induced electrically under general anesthesia, and the heart was excised.

\section{Recording and Pacing Electrodes}

Four plunge needle electrodes were inserted in the basal region of the lateral left ventricular wall, where experimental models have shown there is prominent M-cell-like activity ${ }^{8,9}$ Each 21-gauge stainless steel needle had 8 polyamide-coated tungsten wire electrodes, $50 \mu \mathrm{m}$ in diameter, separated by $1 \mathrm{~mm}$. The most proximal electrode was located approximately $0.5 \mathrm{~mm}$ from the epicardial surface, and unipolar electrograms across the left ventricular walls from epicardial (Epi), mid-myocardial (Mid) and endocardial (Endo) sites were recorded simultaneously. The most proximal and distal plunge electrode sites were used as representative Epi and Endo layers, respectively, and the electrode in between, which recorded the longest activation-recovery interval (ARI) at baseline, was representative of the Mid layer. The ARI was calculated as the interval between the time of minimum first derivative of the intrinsic deflection of the QRS complex and the maximum first derivative of the $T$ wave of unipolar electrograms! 10,11 Previous studies in this experimental model have shown that the ARI from unipolar electrograms closely approximates the local effective refractory period regardless of the T wave morphology?,12

In the present study, the cardiac cycle length was lengthened by vagal stimulation, performed via a $75 \mu \mathrm{m}$ silver wire inserted into the right cervical vagosympathetic trunk ${ }^{8}, 12$ Square pulses, $0.3 \mathrm{~ms}$ in duration and $0.5-1.0 \mathrm{~V}$ in strength, were delivered at a frequency of $20 \mathrm{~Hz}$. The heart was paced from bipolar silver wire electrodes inserted in the right ventricle. Pacing pulses $2.0 \mathrm{~ms}$ in duration were delivered by a programmable cardiac stimulator at twice the diastolic threshold.

\section{Data Acquisition}

In each experiment, 32 transmural unipolar electrograms (8 electrodes $\times 4$ needles) were amplified and filtered at a fixed high-pass setting of $0.05 \mathrm{~Hz}$, and an adjustable lowpass setting of $500 \mathrm{~Hz}$. Analog data were digitized at a sampling rate of $1,000 \mathrm{~Hz}$ (F-tech Co, Ltd, Niigata, Japan). The digitized signals were stored in a personal computer?, 9 The ARI was calculated from each unipolar electrogram, and the ARI dispersion of the ventricle was analyzed as transmural ARI dispersion (ARID), defined as the maximum ARI difference among 8 unipolar electrograms in each needle electrode. Because 4 needle electrodes were inserted in each experiment, 28 data points ( 4 needles $\times 7$ experiments) were available for statistical analysis of ARI in each layer of the ventricle and transmural ARID.

\section{Drug Administration}

To simulate LQT3, anthopleurin-A, dissolved in sterile 


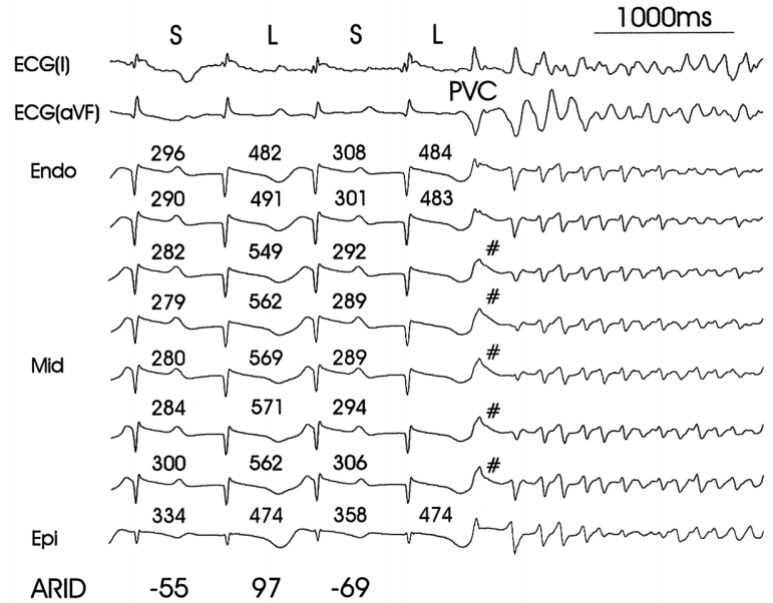

Fig 2. Ventricular tachyarrhythmias (VTA) during T wave alternans (experiment \#1). Surface ECG leads I and $\mathrm{aVF}$ and the transmural unipolar electrograms are shown. A premature ventricular complex (PVC) infringes on the marked spatial dispersion of repolarization of the cycle associated with the longer QT interval, resulting in delayed conduction at the Mid sites (indicated by \#), initiating VTA. Abbreviations as in Fig 1.

saline, was administered as a $5 \mu \mathrm{g} / \mathrm{kg}$ intravenous bolus, followed by a maintenance dose of $0.2 \mu \mathrm{g} \cdot \mathrm{kg}^{-1} \cdot \mathrm{min}^{-1}$. Mexiletine, $2 \mathrm{mg} / \mathrm{kg}$, was administrated intravenously over a few seconds.

\section{Study Protocol and Data Analysis}

The cardiac cycle length was initially increased by onset, then gradually decreased by cessation of electrical vagal stimulation. The vagal stimulation protocol (VSP) has recently been described8 In the present study, the VSP was repeated 8 times before and 5 times after administration of mexiletine in each experiment, and intramural repolarization and activation pattern and the initiation pattern of VTA during heart rate acceleration were analyzed. The ARI and transmural ARID were compared before and after administration of mexiletine during ventricular pacing at a cycle length of $1,000 \mathrm{~ms}$.

\section{Statistical Analysis}

Statistical analysis was performed using Student's t-test, Fisher's exact test and analysis of variance. A $p<0.05$ was considered statistically significant. Values are presented as mean \pm SD.

\section{Results}

\section{Intramural Repolarization Pattern During a Transition Period of Heart Rate Acceleration}

Vagal stimulation induced a slow ventricular escape rhythm (cycle length $800-1,500 \mathrm{~ms}$ ) and prolonged ARI at all sites (Fig 1A). A large transmural ARID was created during bradycardia because of greater ARI prolongation at Mid than at Epi/Endo sites. After cessation of vagal stimulation, the cardiac cycle length gradually shortened to the control level, but intramural unipolar electrograms started to show ARI alternans (Fig 1B). During ARI alternans, the magnitude of ARI oscillation was greater at Mid sites, which was associated with the development of a cycle-tocycle reversed gradient of ventricular transmural repolari-

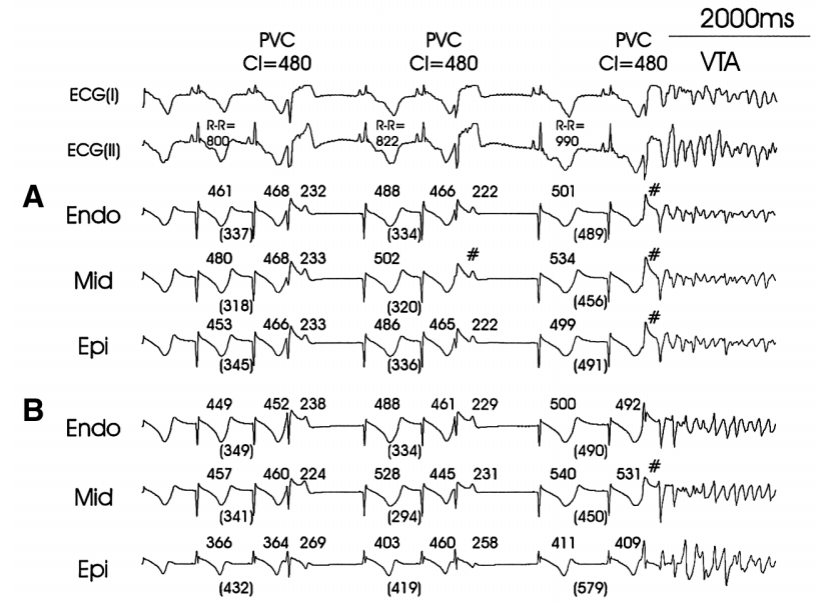

Fig 3. Ventricular tachyarrhythmias (VTA) triggered by premature ventricular complex (PVC) (experiment \#5). Surface ECG leads I and II and selective transmural unipolar electrograms are shown. The unipolar electrograms are from 2 neighboring needle electrodes (Sites A and B) in the left ventricle. During acceleration of the heart rate, frequent PVCs with similar QRS morphology and coupling interval (CI) appear. The last PVC infringes on the prolonged ARI of the preceding sinus beat and creates delayed conduction (indicated by \#) at new sites, initiating VTA. Numbers in each parenthesis indicate diastolic interval. See details in the text. Abbreviations as in Fig 1.

zation. ARI alternans persisted for more than $1 \mathrm{~min}$, then gradually subsided.

\section{Initiation of VTA}

Premature complexes developed during the transition period of heart rate acceleration $(2.2 \pm 2.0$ beats for each VSP, range $0-10$ beats), and VTA were induced in 12 of $56(21 \%)$ attempts of the VSP (Table 1). No VTA was observed after the heart rate had returned to the control level (136 \pm 9 beats/min). Furthermore, as reported previously,,12 bradycardia-dependent spontaneous VTA were also observed in all 7 experiments during vagal stimulation, suggesting an enhanced susceptibility to arrhythmias during this period.

Fig 2 shows surface ECG leads I and aVF, and the transmural electrograms illustrating VTA occurring during ARI alternans in the transition period of heart rate acceleration. A single premature ventricular complex (PVC) infringes on the large spatial dispersion of ventricular repolarization in a cycle with a longer QT interval. Activation of the PVC shows delayed conduction at several Mid sites and triggers the VTA. Fig 3, obtained from a different experiment, shows surface ECG leads I and II, and selective transmural left ventricular electrograms from 2 neighboring needle electrodes. Several PVCs occur after cessation of vagal stimulation and the last one triggers a VTA. The QRS configuration and coupling interval of the PVCs are nearly identical on the surface ECG. However, a longer cycle of $990 \mathrm{~ms}$ between the last 2 sinus events provides a longer preceding diastolic interval for the last cycles. This longer diastolic interval results in a prolonged ARI of the last sinus cycle, such that the last PVC, though its coupling interval is identical to that of previous PVCs, creates new delayed conduction at several Mid/Endo sites and initiates VTA ${ }^{13}$ Unipolar electrograms from 2 needle electrodes show similar intramural activation and repolarization patterns, as the needle electrodes are inserted within a small area of the left ventricular free wall. 


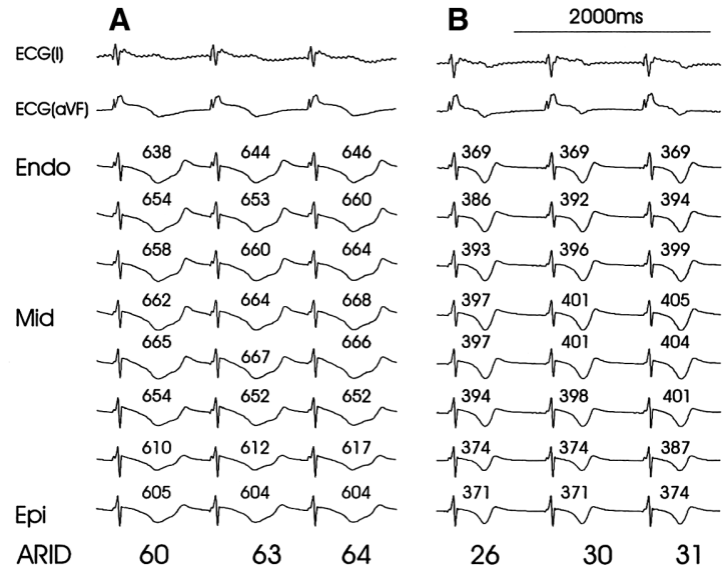

Fig 4. Transmural electrograms before (A) and after (B) mexiletine (experiment \#4). Before mexiletine, ARI at the Mid site is longer than at the Epi/Endo sites and a large ARID of $60-64 \mathrm{~ms}$ is present. All sites show marked ARI shortening after mexiletine administration and ARID decreases to $26-31 \mathrm{~ms}$. Cardiac cycle was fixed at $1,000 \mathrm{~ms}$ by ventricular pacing during vagal stimulation. Abbreviations as in Fig 1.

\section{Antiarrhythmic Effects of Mexiletine}

Mexiletine markedly shortened ARI at all sites (Table 2, Fig 4). Before mexiletine, ARI at Epi was shorter than at Mid/Endo sites, though there was no significant difference in ARI among the 3 layers of the ventricle after drug administration. Mexiletine attenuated ARID from $92 \pm 11$ to $30 \pm 10 \mathrm{~ms}(\mathrm{p}<0.0001)$ at the ventricular paced cycle length of $1000 \mathrm{~ms}$, which was caused by a greater shortening of ARI at Mid/Endo rather than Epi sites (Endo: $-31.0 \pm 9.7 \%$, Mid: $-32.1 \pm 8.8 \%$, Epi: $-24.0 \pm 8.5 \%, \mathrm{p}=0.0019)$. Systolic and diastolic blood pressures decreased non-significantly from $123 \pm 14$ to $112 \pm 10 \mathrm{mmHg}$ and from $63 \pm 8$ to $59 \pm$ $7 \mathrm{mmHg}$, respectively, after mexiletine. Likewise, heart rate was not different before $(136 \pm 9$ beats/min) versus after (130 \pm 12 beats $/ \mathrm{min})$ mexiletine .

After mexiletine administration, the VSP was performed 5 times in each experiment. Neither ARI alternans nor VTA was induced by any of the 35 attempts (12/56 times before vs $0 / 35$ times after mexiletine, $\mathrm{p}<0.003$ ) (Table 1 ). A similar change in heart rate was induced by VSP before and after mexiletine, but only a few premature beats were observed during the transition period of heart rate acceleration after mexiletine $(0.4 \pm 0.7$ complexes in each VSP, range $0-3$ complexes).

\section{Discussion}

\section{Heart Rate Acceleration by VSP}

In previous studies, a faster heart rate associated with sustained sympathetic stimulation decreased transmural dispersion of ventricular repolarization and suppressed VTA in the LQT3 syndrome!4-16 However, abrupt increases in sympathetic activity may have arrhythmogenic effects, 17 because several episodes of VTA in LQT3 syndrome have been observed in association with exercise or emotional stress, and because $\beta$-adrenergic blockade has some protective effects 3,4 To further study the arrhythmogenic effects of heightened sympathetic activity in LQT3 syndrome, clinically relevant experimental conditions were created in the present study by cessation of vagal stimulation such that a state of predominant sympathetic activity was created.
Table 2 ARI and ARID Before and After Mexiletine

\begin{tabular}{lccc}
\hline \hline & Before & After & Befor vs After \\
\hline Endo & $530 \pm 94$ & $360 \pm 46$ & $p<0.0001$ \\
Mid & $555 \pm 101$ & $371 \pm 47$ & $p<0.0001$ \\
Epi & $464 \pm 90$ & $348 \pm 45$ & $p<0.0001$ \\
ANOVA & $p=0.0017$ & $N S$ & \\
ARID & $92 \pm 11$ & $30 \pm 10$ & $p<0.0001$ \\
\hline
\end{tabular}

$n=28$ (4 needles $x 7$ experiments).

ARI, activation-recovery interval; ARID, transmural activation recovery interval dispersion; Endo, endocardial site; Mid, mid-myocardial site; Epi, epicardial site.

It is well known that an abrupt shortening of the cardiac cycle length by rapid pacing can induce repolarization alternans in various experimental conditions 18,19 Attention has been focused on this phenomenon, which is believed to amplify the underlying dispersion of repolarization and represent a precursor of tachyarrhythmias ${ }^{20,21}$ However, in clinical cases, heart rate changes gradually depending on autonomic activity. In the present study, transmural electrograms showed ARI alternans during gradual shortening of the cardiac cycle similar to that observed in rapid pacing models $18,19,22$ and a large spatial and temporal dispersion of ventricular repolarization was created. Different magnitudes of ARI oscillations in each layer of the ventricle were associated with the development of a large transmural dispersion of ventricular repolarization, ${ }^{19,22}$ probably because of differences in restitution kinetics and the diastolic intervals in each layer of the ventricle, resulting in a different restitution pattern within the same cardiac cycle!19,23 As additional arrhythmogenic factors, abnormal automaticity and/or delayed afterdepolarization, enhanced under conditions of predominantly sympathetic activity, seemed to cause VTA-triggering premature beats during heart rate acceleration. Our results suggest that, as during bradycardia,, 12 arrhythmogenesis in the LQT3 model is promoted during the transition period of heart rate acceleration, and suppressed by continued faster, control-level sinus cycle length.

\section{Therapeutic Effects of Mexiletine}

Mexiletine causes a greater QT interval shortening in the LQT3 than in the LQT1 and LQT2 syndromes!,7 Though a shortening of the QT interval may indicate a decreased arrhythmogenic potential in the heart, the correlation between QT interval shortening and protection against VTA has not been firmly established. The VTA of LQTS are usually not predictably reproduced by conventional protocols, including programmed electrical stimulation, $\beta$-adrenergic stimulation and exercise, making comparisons of the degree of inducibility before and after therapeutic interventions unreliable? 24,25 However, in the present study, no VTA was induced by VSP after administration of mexiletine, an observation associated with ARI shortening and attenuation of transmural ARID. Although the inducibility of VTA was not high before mexiletine $(21 \%, 12 / 56$ attempts of VSP), it seems plausible that their complete suppression after mexiletine was attributable to an antiarrhythmic effect of the drug. A decrease in the number of premature beats during heart rate acceleration was also associated with VTA suppression. Previous experimental studies have reported that mexiletine is effective in suppressing abnormal automatic activity of Purkinje fibers ${ }^{26}$ Furthermore, 
mexiletine may counteract the effect of anthopleurin-A, which increases the net inward current, and may explain the decreased number of premature beats after its administration. Although we used the same VSP in all experiments, the number of premature beats and the process of heart rate acceleration were not identical in each attempt, under the same experimental conditions, or across the 7 experiments, probably as a result of variable parasympathetic and sympathetic activity during each episode. The antiarrhythmic effects of mexiletine may have involved, at least in part, inhibition of sympathetic nerve traffic in the ventricle, but the power of sympathetic and parasympathetic activity was not evaluated in each attempt of the VSP, so the detailed mechanism of the development of premature beats and its modification by mexiletine were not entirely clarified by this study.

\section{Clinical Implications}

In some patients with LQT3 syndrome, transient heart rate acceleration caused by an abrupt shift to a state of predominant sympathetic activity increases the heterogeneous distribution of ventricular repolarization and induces triggered premature beats, resulting in the initiation of VTA. Therefore, $\beta$-adrenergic blockade, which can modulate this process of heart rate acceleration and suppress the premature beats, has the potential to be therapeutic in some LQT3 patients. Though it is important to assess the therapeutic effects of $\beta$-adrenergic blockade, unfortunately they were not undertaken in this study. Mexiletine, which homogenizes the distribution of ventricular repolarization and suppresses premature ventricular complexes, may have additional antiarrhythmic effects against VTA during periods of heart rate acceleration.

\section{Study Limitations}

The distribution of M-cells is heterogeneous, such that the limited recording electrodes and small recording area may have underestimated the heterogeneity of ventricular repolarization ${ }^{25}$ Furthermore, the steepest repolarization gradients between the surface of the epicardium and the deep subepicardium are difficult to resolve with unipolar electrograms that begin at a depth of $0.5 \mathrm{~mm}$, a technical limitation that may also underestimate the transmural repolarization gradient ${ }^{27}$

The effects of mexiletine were studied after a single dose. Different doses of mexiletine likely have different effects on VTA inducibility and transmural ARID. The VSP should be attempted 8 times after mexiletine, but we only measured it 5 times. Several mutations have been reported in the genes of LQTS and the clinical characteristics of LQTS vary depending on each mutation ${ }^{28}$ Therefore, experimental studies of drug efficacy using these models are limited in their ability to test the clinical usefulness of therapeutic interventions in all forms of LQTS.

In the present study, we could not measure sympathetic and vagal activity during the transition period of heart rate acceleration. Because heart rate acceleration itself may be associated with arrhythmogenesis during this period. ${ }^{19,22}$ we need to measure the alteration of autonomic tone in future experiments. Spectrum analysis of the body surface ECG may provide some additional information of autonomic activity during the heart rate acceleration period. Moreover, as well as $\beta$-adrenergic stimulation, $\alpha$-adrenergic activity may act arrhythmogenically in the transition period of heart rate acceleration. In congenital LQTS patients, we recently reported beneficial effects of $\alpha$-adrenoceptor blockade 29 Therefore, the effects of both $\beta$ - anda -adrenoceptor blockade on VTA related to the VSP should be studied in future experiments.

Mexiletine appears to have a beneficial effect in LQT3 patients, though its long-term efficacy has not been confirmed in clinical studies. Finally, further studies in a larger study population are required for a detailed analysis of the arrhythmogenic potential of abrupt changes in autonomic activity and the therapeutic effects of mexiletine in the LQT3 syndrome.

\section{References}

1. Zareba W, Moss AJ, Schwartz PJ, Vincent GM, Robinson JL, Priori $\mathrm{SG}$, et al for The International Long-QT Syndrome Registry Research Group. Influence of genotype on the clinical course of the long QT syndrome. N Engl J Med 1998; 339: 960-965.

2. Priori SG, Barhanin J, Hauer RN, Haverkamp W, Jongsma HJ, Kleber AG, et al. Genetic and molecular basis of cardiac arrhythmias: Impact on clinical management part I and II. Circulation 1999; 99: $518-528$.

3. Schwartz PJ, Priori SG, Spazzolini C, Moss AJ, Vincent GM, Napolitano C, et al. Genotype-phenotype correlation in the long QT syndrome: Gene-specific triggers for life-threatening arrhythmias. Circulation 2001; 103: 89-95.

4. Moss AJ, Zareba W, Hall WJ, Schwartz PJ, Crampton RS, Benhorin $\mathrm{J}$, et al. Effectiveness and limitations of beta-blocker therapy in congenital long-QT syndrome. Circulation 2000; 101: 616-623.

5. Zipes DP. The long QT syndrome: A Rosetta stone for sympathetic related ventricular tachyarrhythmias. Circulation 1991; 84: 14141419.

6. Schwartz PJ, Priori SG, Locati EH, Napolitano C, Cantù F, Towbin JA, et al. Long QT syndrome patients with mutations of the SCN5A and HERG genes have differential responses to Na channel blockade and to increase in heart rate: Implications for gene-specific therapy. Circulation 1995; 92: 381-386.

7. Shimizu W, Antzelevitch C. Sodium channel block with mexiletine is effective in reducing dispersion of repolarization and preventing torsade de pointes in LQT2 and LQT3 models of the long QT syndrome. Circulation 1997; 96: 2038-2047.

8. Chinushi M, Hosaka Y, Washizuka T, Furushima H, Aizawa Y. Arrhythmogenesis of T wave alternans associated with surface QRS complex alternans and the role of ventricular prematurity. $J$ Cardiovasc Electrophysiol 2002; 13: 599-604.

9. Chinushi M, Kasai H, Tagawa M, Washizuka T, Hosaka Y, Chinushi $\mathrm{Y}$, et al. Triggers of ventricular tachyarrhythmias and therapeutic effects of nicorandil in canine models of LQT2 and LQT3 syndromes. J Am Coll Cardiol 2002; 40: 555-562.

10. Haws CW, Lux RL. Correlation between in vivo transmembrane action potential durations and activation recovery intervals from electrograms: Effect of interventions that alter repolarization time. Circulation 1991; 81: 281-288.

11. Chinushi M, Washizuka T, Hosaka Y, Furushima H, Tanabe Y, Chinushi Y, et al. Activation-recovery interval as a parameter to assess the intracardiac ventricular repolarization in patients with congenital long QT syndrome. Am J Cardiol 2002; 90: 432-435.

12. El-Sherif N, Caref EB, Yin H, Restivo M. The electrophysiological mechanism of ventricular tachyarrhythmias in the long QT syndrome: Three-dimensional mapping of activation and recovery patterns. Circ Res 1996; 79: 474-492.

13. El-Sherif N, Caref EB, Chinushi M, Restivo M. Mechanism of arrhythmogenicity of the Short-Long cardiac sequence that precedes ventricular tachyarrhythmias in the long QT syndrome. J Am Coll Cardiol 1999; 33: 1415-1423.

14. Priori SG, Napolitano C, Cantu F, Brown AM, Schwartz PJ. Differential response to $\mathrm{Na}$ channel blockade, beta-adrenergic stimulation, and rapid pacing in a cellular model mimicking the SCN5A and HERG defects present in the long-QT syndrome. Circ Res 1996; 78: 1009-1015.

15. Shimizu W, Antzelevitch C. Differential effects of beta-adrenergic agonists and antagonists in LQT1, LQT2 and LQT3 models of the long QT syndrome. J Am Coll Cardiol 2000; 35: 778-786.

16. Tanabe Y, Inagaki M, Kurita T, Nagaya N, Taguchi A, Suyama K, et al. Sympathetic stimulation produces a greater increase in both transmural and spatial dispersion of repolarization in LQT1 than LQT2 forms of congenital long QT syndrome. J Am Coll Cardiol. 2001; 
37: $911-919$.

17. Fujiki A, Nishida K, Mizumaki K, Nagasawa H, Shimono M, Inoue $\mathrm{H}$. Spontaneous onset of torsade de pointes in long QT syndrome and the role of sympathovagal imbalance. Jpn Circ J 2001; 65: 1087 1090.

18. Saitoh H, Bailey JC, Surawicz B. Alternans of action potential duration after abrupt shortening of cycle length: Differences between dog Purkinje and ventricular muscle fibers. Circ Res 1988; 62: 1027 1040.

19. Chinushi M, Restivo M, Caref EB, El-Sherif N. The electrophysiological basis of arrhythmogenicity of QT/T alternans in long QT syndrome: Tridimensional analysis of the kinetics of cardiac repolarization. Circ Res 1998; 83: 614-628.

20. Rosenbaum DS, Jackson LE, Smith JM, Garan H, Ruskin JN, Cohen RJ. Electrical alternans and vulnerability to ventricular arrhythmias. N Engl J Med 1994; 330: 235-241.

21. Pastore JM, Girouard SD, Laurita KR, Akar FG, Rosenbaum DS. Mechanism linking T-wave alternans to the genesis of cardiac fibrillation. Circulation 1999; 99: 1385-1394.

22. Shimizu W, Antzelevitch C. Cellular and ionic basis for T-wave alternans under long QT conditions. Circulation 1999; 99: 1499_ 1507.

23. Laurita KR, Girouard SD, Rosenbaum DS. Modulation of ventricular repolarization by a premature stimulus: Role of epicardial dispersion of repolarization kinetics demonstrated by optical mapping of the intact guinea pig heart. Circ Res 1996; 79: 493-503.

24. Surawicz B. Electrophysiological substrate of torsade de pointes: dispersion of repolarization or early afterdepolarizations? J Am Coll Cardiol 1989; 14: 172-184.

25. Chinushi M, Caref EB, Restivo M, Nollo G, Aizawa Y, El-Sherif N. Cycle length associated modulation of the regional dispersion of ventricular repolarization in a canine model of long QT syndrome. Pacing Clin Electrophysiol 2001; 24: 1247-1257.

26. Arita M, Goto M, Nagamoto Y, Saikawa T. Electrophysiological actions of mexiletine on canine purkinje fibers and ventricular muscle. Br J Pharmacol 1979; 67: 143-152.

27. Yan GX, Shimizu W, Antzelevitch C. Characteristics and distribution of $\mathrm{M}$ cells in arterially perfused canine left ventricular wedge preparations. Circulation 1998; 98: 1921-1927.

28. Satler CA, Vessely MR, Duggal P, Ginsburg GS, Beggs AH. Multiple different missense mutations in the pore region of HERG in patients with long QT syndrome. Hum Genet 1998; 102: 265-272.

29. Furushima H, Chinushi M, Washizuka T, Aizawa Y. Role of a 1blockade in congenital long QT syndrome: Investigation by exercise stress test. Jpn Circ J 2001; 65: 654-658. 